\title{
Non-invasive Diagnosis in Clinically Suspected ASD Secundum
}

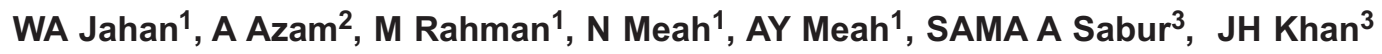 \\ ${ }^{1}$ Department of Radiology and Imaging, NICVD, ${ }^{2}$ Department of Cardiology, NICVD, \\ ${ }^{3}$ Department of Cardiac Surgery, NICVD. Dhaka.
}

Keywords:
Atrial Septal
Defect,
Cardiac
Imaging

\begin{abstract}
:
Background: The non-invasive tests like X-ray, ECG and Echocardiography are viewed as an extension of clinical art in cardiology and have become an integral part of history taking, physical examination and other diagnostic method.Atrial Septal Defect (ASD) of secundum type is defined as a through and through communication at atrial level. Previously the diagnosis and decision of surgery for ASD, mandatorily advocate cardiac catherization.Now cardiologist and cardiac surgeon very hardly asked for cardiac catheterization. Non-invasive diagnosis with the help of ECG, X-ray and Echo is sufficient for its diagnosis and treatment for surgery. In Bangladesh there is no study upon it. Considering this ground the study is perform on Bangladeshi patients.
\end{abstract}

Methods: Forty six consecutive patients with clinical (auscultatory and electrocardiographic) signs of uncomplicated atrial septal defect of secundum type were examined by chest x-ray, ECG and echocardiography, before right heart catheterisation.

Result: Thirty four (74\%) had ASD, four patients (9\%) had insignificant pulmonary stenosis, and eight subjects (17\%) were normal. No false positive diagnosis of atrial septal defect was made by chest $x$-ray examination, whereas increased vascular markings were incorrectly interpreted as pulmonary congestion in one case. Eight patients had $x$-ray films showing questionable signs of left-to-right shunt. Twelve of 30 patients with a large left-to-right shunt were correctly selected for surgery based on radiological findings.

Conclusion: Analysis of non invasive diagnosis and management of ASD secundum conform the usually described pattern in western literature.

(Cardiovasc. j. 2010; 2(2) : 223-226)

\section{Introduction:}

Forty six consecutive patients with clinical (auscultatory and electrocardiographic) signs of uncomplicated atrial septal defect of secundum type were examined by chest x-ray, ECG and echocardiography, before right heart catheterisation. Thirty four (74\%) had ASD, four patients (9\%) had insignificant pulmonary stenosis, and eight subjects $(17 \%)$ were normal. No false positive diagnosis of atrial septal defect was made by chest x-ray examination, whereas increased vascular markings were incorrectly interpreted as pulmonary congestion in one case. Eight patients had x-ray films showing questionable signs of left-to-right shunt. Twelve of 30 patients with a large left-to-right shunt were correctly selected for surgery based on radiological findings. Echocardiographic distinction between those with atrial septal defect and those without atrial septal defect was correct in all cases; quantitative measurement of left-to-right shunt, however, was unsatisfactory. Combined normal findings by x-ray film and echocardiography appeared adequate in all cases for the exclusion of atrial septal defect (twelve patients). When the twelve

Patients who were correctly identified for surgery from the radiological findings are included, there was a total of 24 patients out of $46(52 \%)$ who were evaluated definitively by the non-invasive tests.

The patient with fixed splitting of the second heart sound on auscultation, incomplete right bundle branch block, and a normal electrocardiographic QRS axis frequently presents a diagnostic problem. Though suspicion of an atrial septal defect of secundum type in such patients will often prove justified, heart catheterisation in others will disclose no signs of atrial septal defect or any other structural abnormality. New techniques such as two-dimensional contrast echo-cardiography, ultrasonic Doppler examination and radionuclide 
methods may become future routine examinations in suspected atrial septal defect. At present, however, chest x-ray examination, phonocardiography, and M-mode echocardiography are well established noninvasive techniques readily available in most cardiac centers. The purpose of the present study was to investigate the reliability of these techniques in the setting of clinically suspected uncomplicated of the atrial septal defect.

\section{Patients and methods:}

During a one year period 46 consecutive patients with a clinical suspicion of atrial septal defect were, referred for heart catheterisation. There were 28 male and 9 female patients, range 5 to 48 years, mean 15 years. The patients were defined by a fixed - splitting of the second heart sound on auscultation accompanied by a grade 1-2/6 systolic ejection murmur in the pulmonary area and incomplete or complete right bundle-branch block with a normal QRS axis. Patients suspected of pulmonary hypertension or structural abnormali-ties other than atrial septal defect were excluded from the study, based on physical examination and electrocardiogram. The day before heart catheterisation non-invasive examinations including chest $\mathrm{x}$-ray, and M-mode, 2DE and Color Doppler echo-cardiography were carried out and evaluated separately by three different observers. Each examiner was unaware of the results of the two other tests.
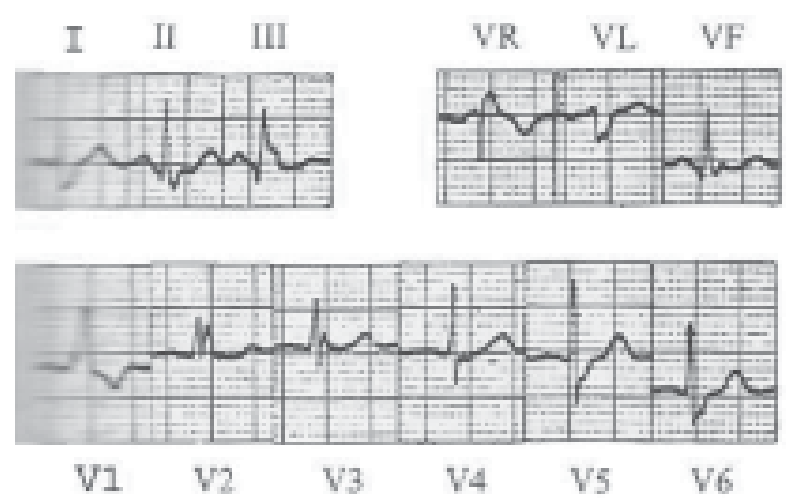

Fig.-1: RBBB in ASD secundum

Chest $x$-ray examination was performed in the erect position in the posteroanterior and lateral projections. Signs of increased pulmonary blood flow in patients with fixed splitting of the second heart sound and incomplete right bundle-branch block were considered diagnostic of atrial septal defect. Thus, patients with intrapulmonary vessels in the upper lung zones of increased calibre and readily visible to the peripheral edge of the lungs were classified as having atrial septal defect. Atrial septal defect requiring operation was diagnosed in those cases with intrapulmonary vessels of increased calibre in all lung zones. Equivocal findings were denoted as questionable atrial septal defect or questionable atrial septal defect requiring surgery, respectively.

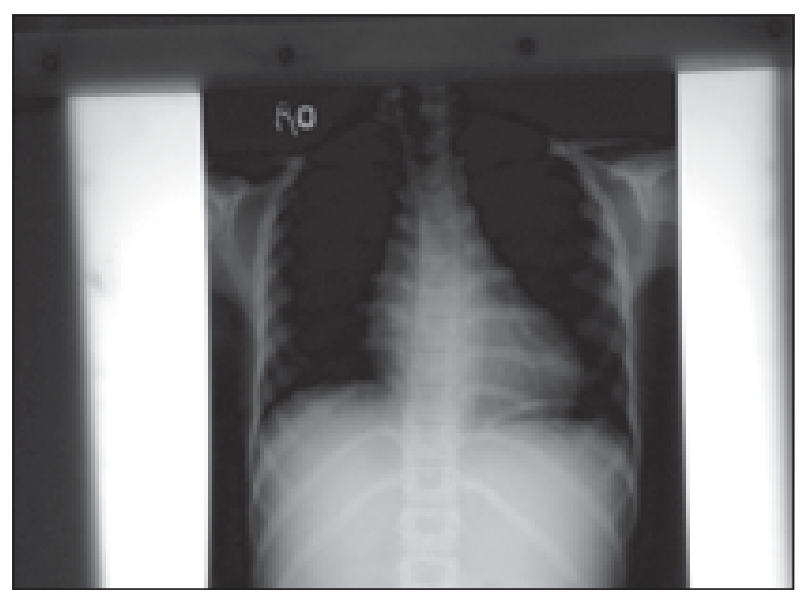

Fig- 2: $X$-ray Chest $P A$ view in $A S D$ secundum

Echocardiography was recorded using Standared Echo mechines from apex to base were performed in the supine and left lateral positions with the transducer in the third, fourth, and fifth left intercostal spaces near the sternal border. Right ventricular end-diastolic dimension was measured in the supine position at the chordal level according to Feigenbaum using the intercostal space with the largest right ventricular end-diastolic dimension (RVD). Echo drop out on IAS having mosaic signal across it from L-R, paradoxical movement of IVS and right sided volume overload with dilated RA,RV and PA was regarded as diagnostic of atrial septal defect. An M-mode and Doppler scan from the tricuspid to the mitral valve was performed in each inter-costal space in the supine and left lateral positions to see the tricuspid flow. 


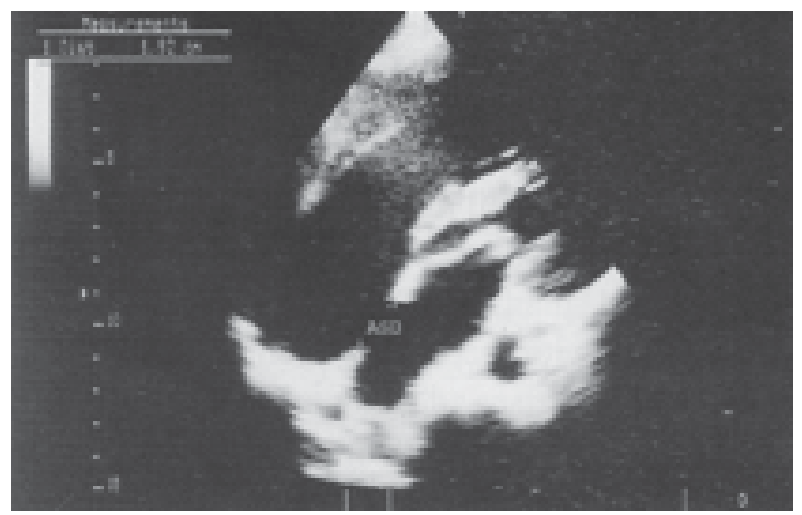

Fig.-3: $2 D E$ echo in ASD secundum

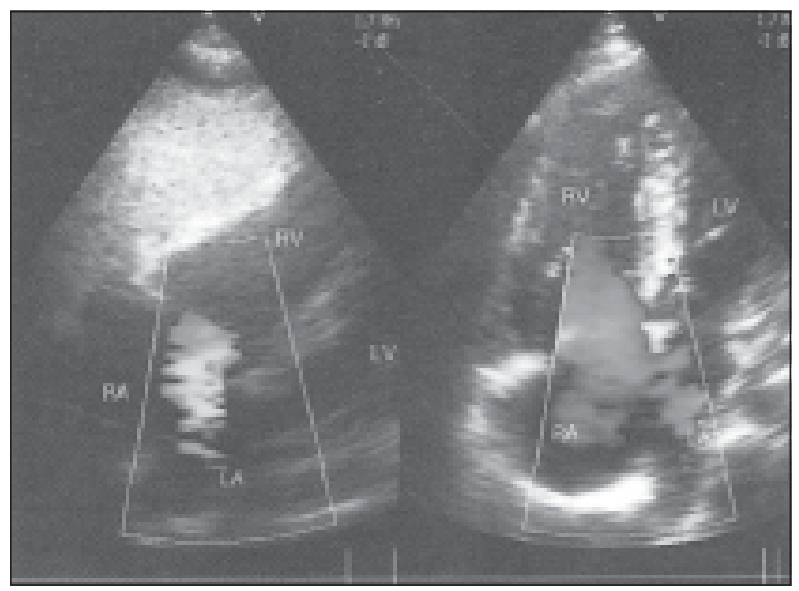

Fig.-4: Color Doppler Echo in ASD secundum

Cardiac catheterisation was performed via the right femoral vein. Pulmonary-to-systemic flow ratio was calculated by the oximetric method. If no left-toright shunt was demonstrable on oximetry the atrial septum was carefully probed to exclude a communication (four cases) Patients with a flow ratio equal to or larger than 2.0 or a flow ratio larger than 1.8 in combination with cardiac enlargement were referred for operation.

\section{Results:}

A left-to-right shunt at atrial level was shown in 34 patients $(74 \%)$ while 12 patients $(26 \%)$ had no detectable atrial septal defect. The left-to-right shunt was small in four patients (flow ratio $=1.3$ and 1.5 , respectively). The six patients without atrial septal defect included four with normal findings at right heart catheterisation and two patients with negligible valvular pulmonary stenosis

\section{Chest X-ray:}

No false positive diagnosis of left-to-right shunt was made on chest X-ray. An increased flow pattern in a 48-year-old man was erroneously interpreted as pulmonary congestion. Eight cases with flow ratios ranging from 1-5 to more than 4 were classified as having questionable signs of leftto-right shunt, whereas a left-to-right shunt was predicted in a patient with a flow ratio of only 1-3. 12 patients out of 24 with definite radio-logical signs of left-to-right shunt were correctly suggested as needing operation according to our present guidelines. The other 12 patients in-cluded 6 with questionable signs of a large shunt and 6 patients who were classified as having atrial septal defect not requiring operation, but, of the last-mentioned, 4 patients had flow ratios of more than 2.0 and were in fact referred for operation.

\section{Discussion:}

Though right heart catheterisation in older children and adults is a relatively simple procedure carrying only a minimal risk, it would of course be desirable to avoid this examination in healthy subjects with a clinical suspicion of atrial septal defect. Moreover, non-invasive identification of surgical candidates would save time and reduce costs. In our series, chest x-ray examination appeared satisfactory in excluding left-to-right shunt. Only one false nega-tive interpretation was made. Increased vascular markings in this case were erroneously regarded as pulmonary congestion; the patient, however, would of course not have been acquitted of heart disease on the basis of the radiological findings. Furthermore, combination of the radio-logical findings with echocardiography would immediately have corrected the radiological diagnosis.

No false positive radiological diagnosis was suggested, but only questionable signs of left-toright shunt were described in 8 out of 34 patients with atrial septal defect including 6 patients with flow ratios of $2.1,2.7$, and more than 4 , respectively. Radiological markings suggesting need for operation seemed reliable; however, only 12 out of 30 such patients were identified. The radio-logical suggestion of a small atrial septal defect, on the other hand, correlated poorly with catheterisa-tion data; 2 patients was correctly regarded as having a small shunt, whereas 4 patients with large shunts were erroneously classified as having small atrial septal defects not requiring operation. 
Echocardiography distinguished between un-complicated atrial septal defect and non-atrial septal defect. Occasional reports have shown the occa-sional appearance of a patient with atrial septal defect and a normal echocardiogram. These cases were all patients with small left-to-right shunts (flow ratio less than 2.0) One should, however, be aware of a possible false negative echocardiogram. Moreover, echocardiograms indicating right ven-tricular volume overload in 4 normal patients have been reported. Thus, the addition of suppor-tive information from ECG and chest $\mathrm{x}$-ray examination is advisable.

The considerable variation in type of septal motion within the same patient is noteworthy. This finding has been described by Kerber et al in occasional patients, but appears from our series to be a common feature in patients with atrial septal defect; the degree of variation is probably dependant on the number of projections available.

\section{Conclusion:}

Given an auscultatory and electrocardiographic suspicion of uncomplicated atrial septal defect, the combined finding of a normal chest x-ray film and a normal M-mode, 2DE and Color Doppler echocardiogram appears to indicate that there is no atrial septal defect. Confirmation of an atrial septal defect was correctly obtained by echocardiography in all our cases and no false positive diagnosis was made either by radiological examination. Our data on small atrial septal defects with left-to-right shunts below the opera-tive range are too limited to draw definite conclu-sions. It appears that a patient with echocardio-graphic findings of right ventricular volume over-load, and a chest x-ray suggesting a large left-to-right shunt will, at right heart catheterisa-tion, demonstrate the need for closing an atrial septal defect.

In our series of 46 patients with a clinical suspicion of atrial septal defect all 12 patients without defects were correctly excluded by chest $\mathrm{x}$-ray and echocardiography. 12 patients with atrial septal defect diagnosed by chest $\mathrm{x}$-ray and echocardiographic con-firmation in four cases were correctly selected for operation based on radiological findings. Thus, a total of 24 out of 46 patients (52\%) appear to be definitely evaluated without heart catheterisation.
Improved non-invasive diagnostic results can perhaps be provided in the future using techniques such as radionuclide examinations and by develop-ing ultrasonic methods.

\section{Reference:}

1. Fuster V, Brandenburg RO. McGoon DC et al. Clinical approach and management of congenital heart disease in the adolescent and adult. Cardiovasc Clin 1980; 10: 15.

2. Grenadier E, Alpam G, Keidar S et al. M-mode and two dimensional echocardiography in adult patients with atrial septal defects. Clin. Cardiol 1983; 6:588.

3. King E ME. Atrial septal defects : Echocardiographic evaluation. In: The practice of clinical echocardiography $2^{\text {nd }}$ ed. Philadelphia, WB Saunders, 2002: 881.

4. Mugge A, Daniel WG, Angermann C et al. Atrial septal aneurysm in adults patients. A multicentre study using transthoracic and transoesophageal echocardiography. Circulation 1995; 91: 2725.

5. Pollack C, Sullivan H, Lujec B et al. Doppler color flow imaging assessment of shunt size in ASD. Circulation 1988; 78: 522-528.

6. Yock PG, Popp RL. Non invasive estimation of RVSP by Doppler ultrasound in patients with tricuspid regurgitation. Circulation 1984; 70: 657.

7. Callahn MJ, Seward JB, Tajic AJ. Two dimensional and Doppler echocardiography in atrial septal defect: Operation without catheterization. Echocardiography 1984; 1: 521-526.

8. Dittman H, Jacksch R, Voelker W et al. Accuracy of Doppler echocardiography in quantitation of left to right shunt in patients with atrial septal defects. $J$ Am Coll Cardiol 1988; 11: 338-342.

9. E King E ME. Echocardiographic evaluation of the adult with unoperated congenital heart diseases. In: The practice of clinical echocardiography $2^{\text {nd }}$ ed. Philadelphia, WB Saunders, 2002: 879.

10. Gatzoulis MA, Redington AN, Somervile J et al. Should atrial septal defect in adult be closed? Ann Thrac Surg 1996: 61; 657-9.

11. Horath KA, Burke RP, Collins JR et al. Surgical treatment of adult atrial septal defect: early and long term result. J Am Coll Cardiol 1992; 20: 1156-9.

12. Kitabatake $A$, Inoqe $M$, Asao $M$ et al. Non invasive evaluation of the ratio of pulmonary to systematic flow in atrial septal defect by duplex Doppler Echo. Circulation 1984; 69: 73-79.

13. Stewart WJ, Jiang L, Mich R et al. Varriable effects of changes in flow rate through the aortic, pulmonic and mitral valves on area and flow velocity. Impact on quantitative Doppler flow calculation. J Am Coll Cardiol 1985; 6: 653 . 